\title{
Organized Representation of Spectrotemporal Features in Songbird Auditory Forebrain
}

\author{
Gunsoo Kim and Allison Doupe \\ Department of Physiology and Psychiatry, University of California San Francisco, San Francisco, California 94143
}

Much of our understanding of the functional organization of auditory cortex is based on relatively simple stimuli, and the cortical representation of complex sounds remains poorly understood. With their rich vocal communication and learning behaviors, songbirds can offer insights into the neural processing of complex acoustic signals analogous to human speech. In the primary forebrain auditory area field L (primary auditory cortex analog) of zebra finches, previous studies identified a limited set of spectrotemporal receptive field (STRF) types, but whether these were spatially organized remained unclear. Here, we investigated the spatial organization of field L in multiple anatomical planes, using multielectrode array recordings and a stimulus that captures aspects of spectrotemporal modulations of song. Our data demonstrate two separate axes along which spectrotemporal aspects of sound are mapped: width of spectral tuning changes systematically from narrow to broad mediolaterally, whereas width of temporal tuning changes markedly, from narrow to broad, from the input to the output layers. These spatial patterns, which are evident in STRFs computed from both multiple and single units, result in a mapping of basic STRF types to subregions of field L. This highly organized representation of spectrotemporal features of sound contrasts with current views of mammalian auditory cortex, where no strong spatial organization of STRF shapes has been seen thus far. Our data identify a coherent initial cortical representation of sound features and suggest that forebrain filtering for basic perceptual qualities of sound occurs in a spatially organized and segregated manner in the songbird auditory system.

\section{Introduction}

How natural stimuli such as human speech or birdsong would be represented across the multiple maps for various acoustic parameters found in mammalian auditory cortex remains a challenge (Schreiner et al., 2000; Schreiner and Winer, 2007). In contrast to visual cortex, there is little evidence for stepwise and localized representation of fundamental stimulus features in auditory cortex (Hubel and Wiesel, 1962; Ferster et al., 1996; King and Nelken, 2009). Reverse correlation techniques using complex sounds have revealed diverse types of spectrotemporal receptive fields (STRFs) (Eggermont et al., 1983; Kowalski et al., 1996a,b; Depireux et al., 2001; Miller et al., 2002). Although STRF properties may vary across cortical layers (Atencio et al., 2009), the spatial organization of these cortical STRFs remains elusive. Without knowing the spatial organization of STRFs, however, it is difficult to understand how feature sensitivity might arise through cortical circuitry.

The songbird auditory system offers a valuable additional model for studying the neural representation of complex sounds.

\footnotetext{
Received April 20, 2011; revised Oct. 3, 2011; accepted 0ct. 5, 2011.

Author contributions: G.K. and A.D. designed research; G.K. and A.D. performed research; G.K. analyzed data; G.K. and A.D. wrote the paper.

This work was supported by NIH Grants MH55987 and RC2NS069350 and a Human Frontier Science Program grant to A.D. We thank Brian Wright and Kathy Nagel for help in the initial stages of experiments; Adria Arteseros for technical assistance; and Raghav Rajan, Mimi Kao, Helen McLendon, Michael Brainard, Craig Atencio, and Christoph Schreiner for comments on this manuscript and helpful discussions. We also thank Ken Soderstrom for generously providing the CB1 antibody.

Correspondence should be addressed to Allison Doupe, 513 Parnassus Avenue, Box 0444, Department of Physiology, University of California San Francisco, San Francisco, CA 94143-0444. E-mail: ajd@phy.ucsf.edu.

DOI:10.1523/JNEUROSCI.2003-11.2011

Copyright $\odot 2011$ the authors $\quad 0270-6474 / 11 / 3016977-14 \$ 15.00 / 0$
}

Like humans, songbirds learn their vocalizations using hearing and show lifelong perceptual learning of other birds' songs. They also possess a discrete hierarchy of cortical auditory areas. The equivalent of primary auditory cortex (A1) in the songbird telencephalon is the primary auditory area field L (Karten, 1968; Fortune and Margoliash, 1992; Wild et al., 1993; Dugas-Ford and Ragsdale, 2009; Wang et al., 2010). Field L receives direct inputs from auditory thalamus (ovoidalis; see Fig. $1 \mathrm{~A}, \mathrm{Ov}$ ), primarily into its input layer, L2, which is analogous to layer 4 of A1 (Vates et al., 1996). Layer L2 projects to output layers L1 and L3, which in turn project to higher auditory areas (see Fig. $1 A$ ).

Pure tone stimulation revealed tonotopic organization in field L (Scheich et al., 1979; Heil and Scheich, 1985; Müller and Leppelsack, 1985; Gehr et al., 1999; Terleph et al., 2006), and the identification of field L STRFs further elucidated how complex sounds are encoded (Theunissen et al., 2000; Sen et al., 2001; Woolley et al., 2005, 2009; Nagel and Doupe, 2008). Interestingly, there is a relatively limited set of basic STRF types that have been related to different perceptual qualities such as rhythm, pitch, and timbre (Nagel and Doupe, 2008; Woolley et al., 2009). In addition, mapping studies in songbirds suggested differences in temporal processing across layers (Hose et al., 1987; Nagel and Doupe, 2008). It has remained unclear, however, whether the entire field L complex is organized in terms of the spectrotemporal features revealed by complex stimuli.

Here, we investigated the spatial organization of STRFs in zebra finch field L using a stimulus with rich spectrotemporal modulations. Our results demonstrate a dramatic layer dependence of temporal tuning and a mediolateral gradient of spectral tuning within each field L layer. Furthermore, we could map basic 
A

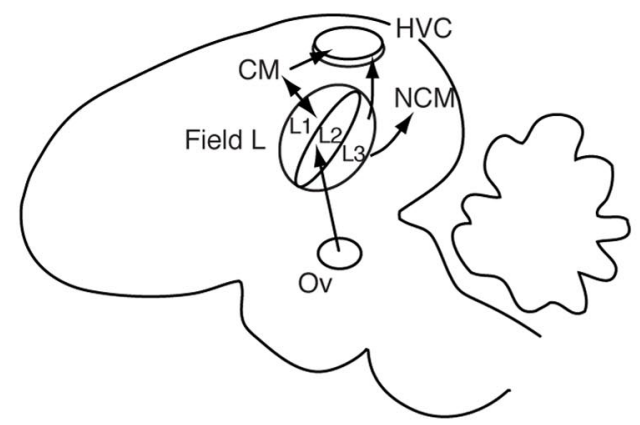

B

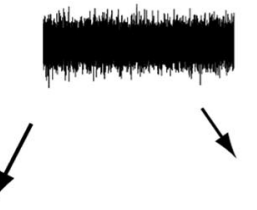

Gaussian white noise

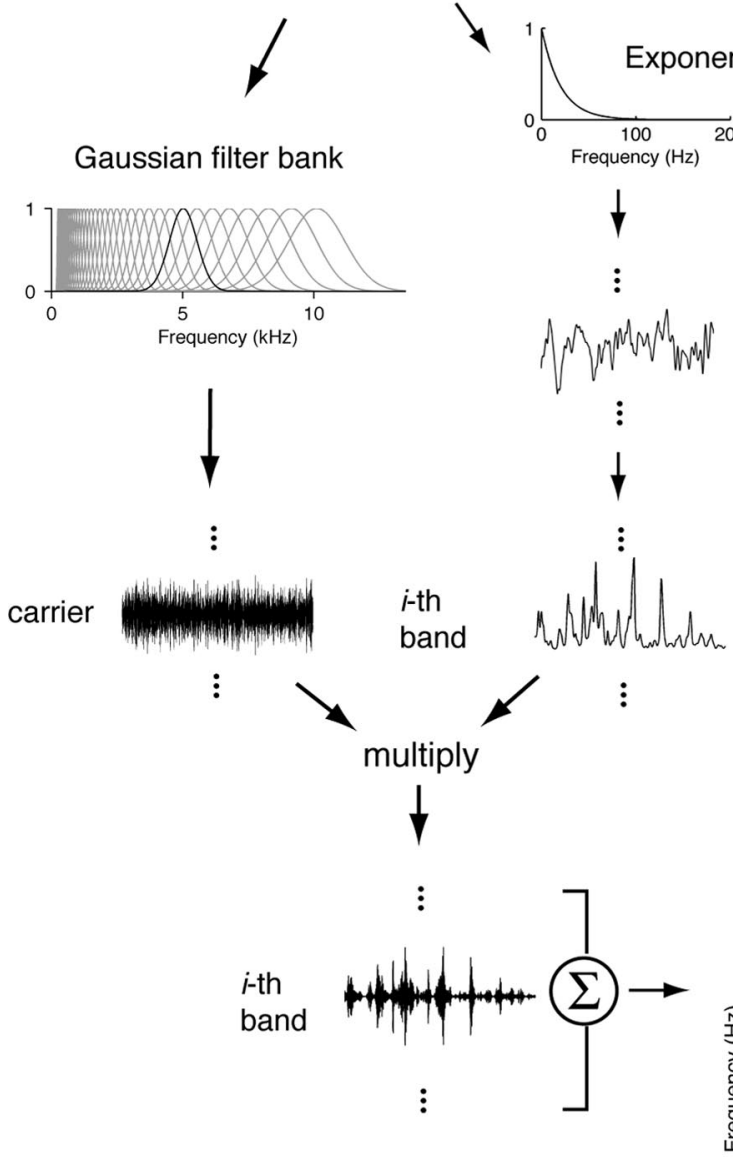

$\log (A M)$
C Nissl

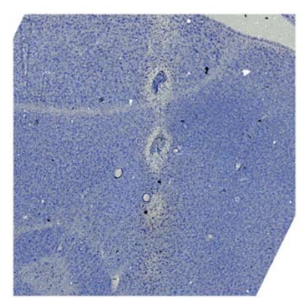

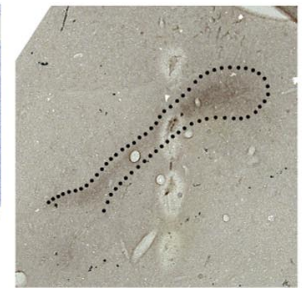

Figure 1. Schematic of the songbird brain and the stimulus design. $\boldsymbol{A}$, Sagittal view of songbird auditory forebrain. NCM, Caudomedial nidopallium; 0v, ovoidalis; HVC, used as a proper name. $\boldsymbol{B}$, Stimulus generation. Left column, The carrier of the stimulus is obtained by filtering white noise using a bank of Gaussian filters whose center frequencies are logarithmically spaced (gray). Right column, The log amplitude envelope of the stimulus is obtained by filtering white noise with an exponential filter (time constant, $20 \mathrm{~Hz}$ ). Each carrier frequency band (black) was multiplied by an exponentiated log envelope to generate a series of amplitude-modulated (AM) signals. This entire series of signals at different frequency bands was summed to yield our final stimulus. C, Examples of field L histology. Adjacent parasagittal sections stained for Nissl (left) or CB1 (right) are shown. The L2 layer types of STRFs onto subregions of field L. Our results indicate that spectrotemporal modulations are processed in a spatially organized manner in the auditory forebrain of a vocal learner and raise the question of whether such organization exists more generally in animals with complex acoustic communication.

\section{Materials and Methods}

Electrophysiology. We mapped field L in 14 adult ( $>120$ posthatch days) male zebra finches (Taeniopygia guttata) raised in our colony. All procedures were performed in accordance with protocols approved by the University of California, San Francisco Institutional Animal Care and Use Committee. Birds were deprived of food and water for $1 \mathrm{~h}$ and anesthetized with three injections of $25 \mu \mathrm{l}$ of $20 \%$ urethane, a cortically sparing anesthetic. The head of the bird was securely held in a custom-made stereotaxic head holder, and a metal post was attached to the skull using dental cement. Once the cement dried, the head was secured by the metal post in a stereotaxic frame. Throughout the experiments, birds lay on an electric heating pad with continuous temperature control (FHC). A linear array of eight tungsten electrodes separated by $190-240 \mu \mathrm{m}$ (impedance, 3-5 M $\Omega$; $75 \mu \mathrm{m}$ shank diameter; FHC) was positioned over the left field $\mathrm{L}$ at appropriate coordinates for the planes of mapping. For a transverse plane, the electrode array was oriented mediolaterally, with the most medial electrode at $0.6 \mathrm{~mm}$ from the midline and $1.5 \mathrm{~mm}$ anterior to the reference point, the bifurcation of the sagittal sinus. For the three parasagittal planes, the array was oriented rostrocaudally and placed at $0.7,1.2$, or $1.8 \mathrm{~mm}$ from the midline, with the most anterior electrode at $2.2 \mathrm{~mm}$ rostral to the reference point. An $\sim 1 \times 2 \mathrm{~mm}$ window was opened in the skull, and the dura was removed. After the electrode array was placed in the brain, a silicone elastomer was applied around the opening to prevent drying.

Neural recordings were performed in a double-walled sound-attenuation chamber. Sound stimuli were played at $70 \mathrm{~dB}$ SPL through a speaker (JBL Scientific Biotechnology Services) located $20 \mathrm{~cm}$ away from the bird, and the sound level was calibrated using a sound level meter (A-weighting; Brüel and Kjær). Neural data were acquired using a headstage (HS-36) and amplifiers (Lynx-8) from Neuralynx and custom software (Multikrank; written by D. Schleef and modified for multichannel recording by B. D. Wright). Electrode arrays were advanced in 200 or $250 \mu \mathrm{m}$ steps, and neural activity was recorded at $8-10$ depths. At the end of each mapping experiment, lesions were made through the first,

is labeled with a dotted line in the CB1-stained section, and lesion sites along the electrode tract are also evident in both sections. 
A
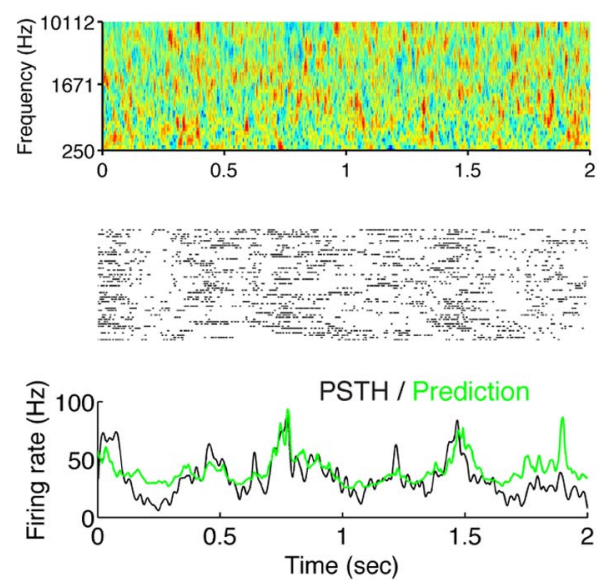
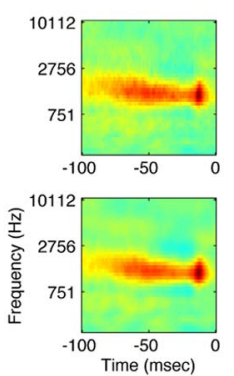

STA<smiles>[3H][3H]</smiles>

STRF
B
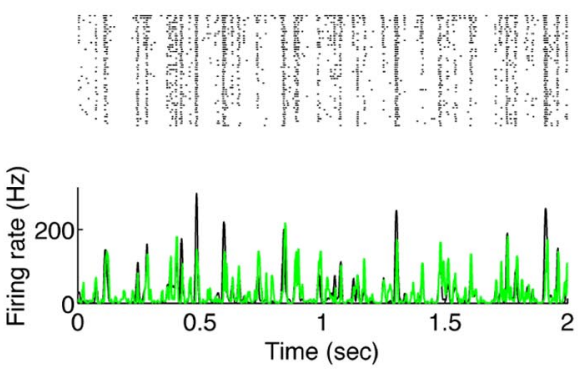

D
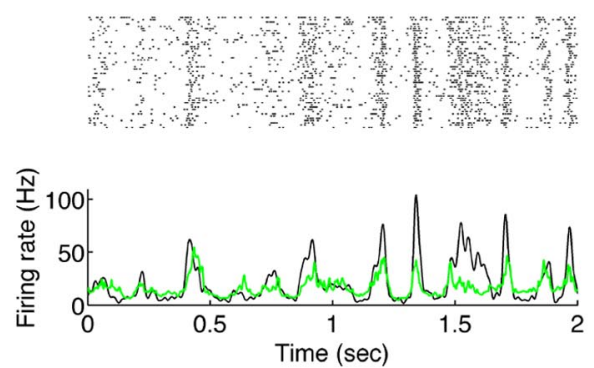

L3
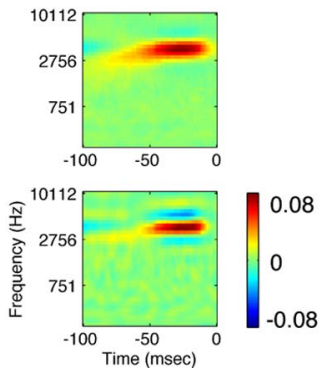

C
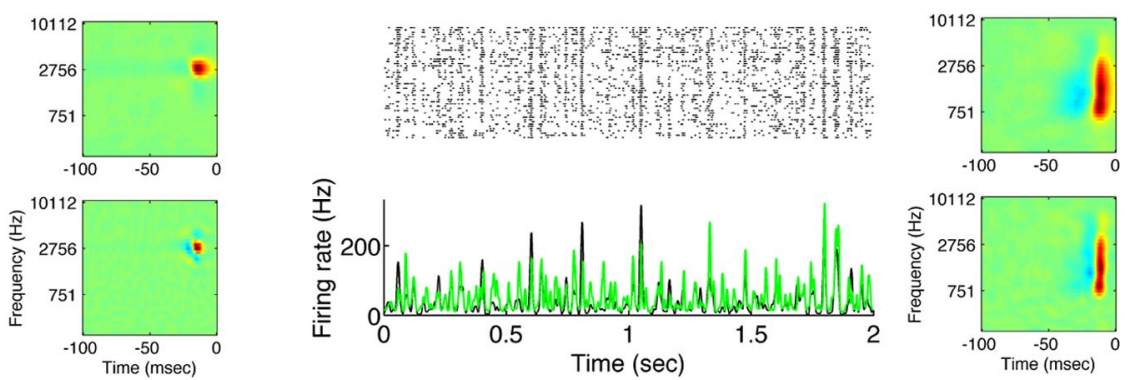

E

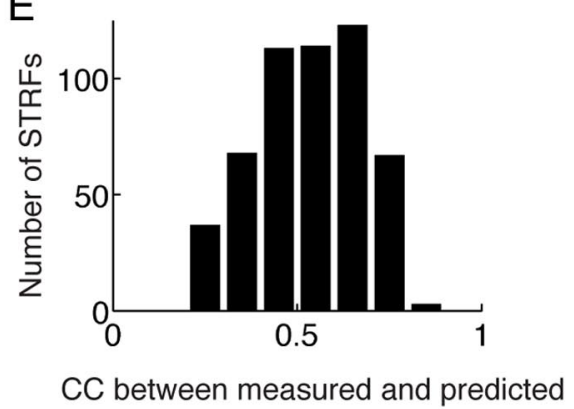

Figure 2. Examples of responses and STRFs at sites in different field L layers. A-D, Spike raster plots and PSTHs from different subregions of field $L$ are shown (left). The stimulus is shown at the top in $\boldsymbol{A}$; the segment shown is a portion of the stimulus that was repeated 50 times. The corresponding STAs and STRFs are also shown (right). Spike raster plots for repeated trials, PSTHs (black), and predicted firing rates (green) are shown to illustrate the response properties of different regions and the prediction quality of the STRFs, but the STAs were computed from the unrepeated, continuously changing part of the stimulus (see Materials and Methods). Also note that removing the stimulus correlation from the STAs sharpened the features, but because of the stimulus design, the basic shapes were preserved in the final STRFs (see Materials and Methods). The sites are from L1 (A), medial L2 (B), lateral L2 (C), and L3 (D). The same 5 s stimulus was played in all sites shown, and the spectrogram of a portion of the stimulus is shown in $\boldsymbol{A}$. The C( values between the measured and predicted firing rates for the example sites shown are $0.57(\boldsymbol{A}), 0.64(\boldsymbol{B}), 0.65(\boldsymbol{C})$, and 0.72 (D). $\boldsymbol{E}$, Distribution of prediction CC values for all 525 mapped sites [0.53 (mean) \pm 0.14 (SD); range, $0.2-0.82$ ].

fourth, and eighth electrodes of the array ("first" is either most medial or most rostral) at three to four depths separated by $500 \mu \mathrm{m}(7.5 \mu \mathrm{A}$ for $10 \mathrm{~s})$. Birds were transcardially perfused with lactated Ringer's solution followed by $3.7 \%$ formaldehyde. Parasagittal $40-\mu \mathrm{m}$-thick sections were cut on a freezing microtome, and alternating sections were processed for Nissl or type 1 cannabinoid receptor (CB1) antibody staining. The CB1 staining strongly labels the L2 layer (Soderstrom et al., 2004; Fig. 1C).

Stimulus generation and STRF calculation. We used a synthetic stimulus similar to that used in a previous study (Fig. $1 B$; Nagel and Doupe, 2008). A series of carrier frequency bands was obtained by filtering Gaussian white noise with a Gaussian filter bank (Fig. $1 B$, left column). The center frequencies of the filters were given by $\exp (\log (250)+0.1(i-$ $1)$ ), where $i$ is the band number (1-38), and the separation between neighboring center frequencies was set to $1 \mathrm{SD}$ to ensure sufficient overlap (Theunissen and Doupe, 1998). Each carrier band was amplitude modulated by envelopes generated by filtering Gaussian white noise with an exponential filter. The filter had a decay constant of $20 \mathrm{~Hz}$ and was designed to mimic the preponderance of lower temporal frequencies in natural sounds (Singh and Theunissen, 2003). After filtering [Fig. $1 B$; $\log (\mathrm{AM})$ ], the signal was exponentiated (Fig. $1 B, A M)$. The final stimulus was generated by adding up all the randomly amplitude-modulated bands (Fig. $1 \mathrm{~B}$, bottom row). For STRF generation, a long, unrepeated sequence of this stimulus was played ( $25 \mathrm{~min}$ ). In addition, a $5 \mathrm{~s}$ segment was repeated 50 times for estimation of the prediction quality of STRFs (see below).

We calculated STRFs using previously described procedures (Theunissen et al., 2000; Nagel and Doupe, 2008). To obtain the spectrotemporal 


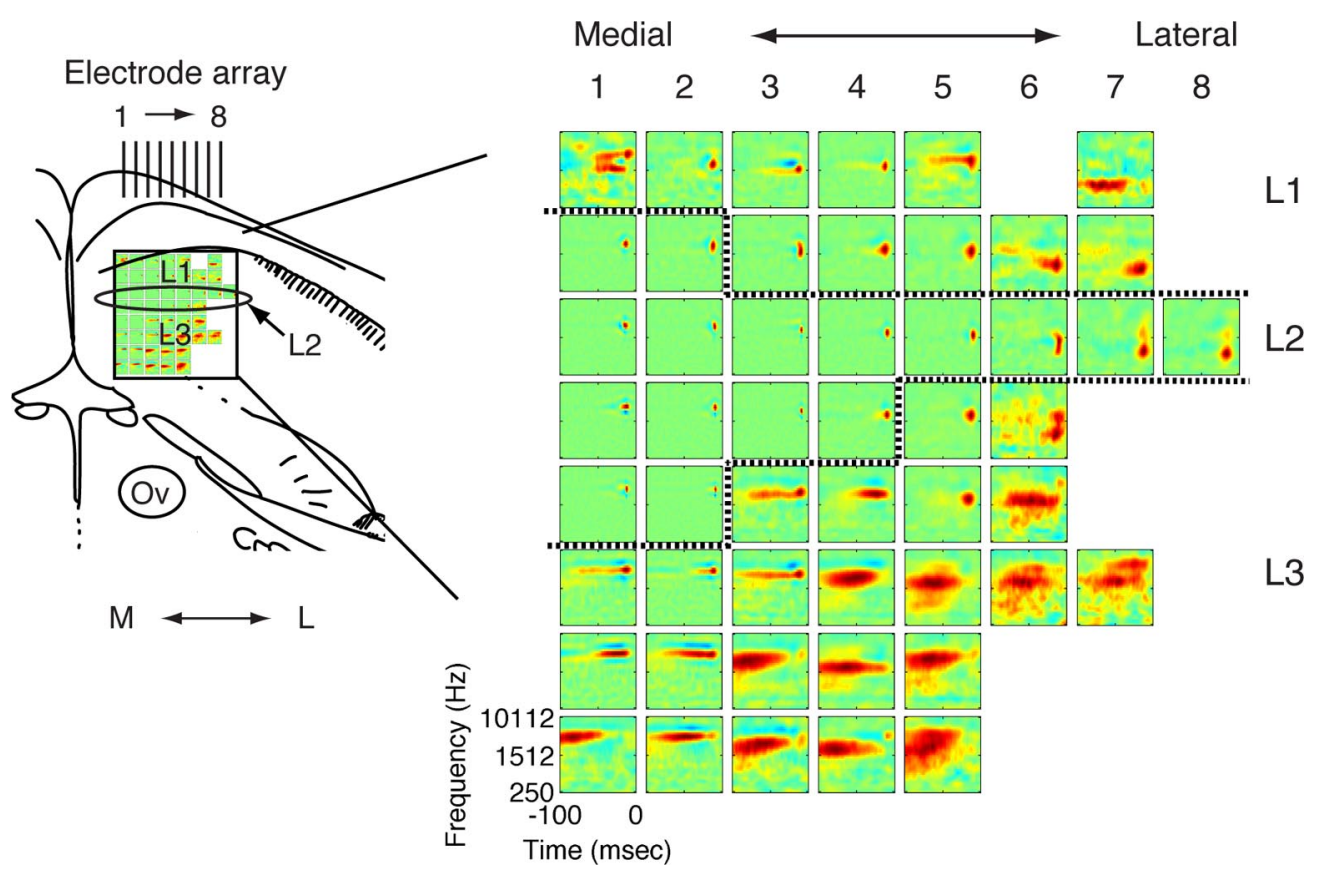

Figure 3. Example map of STRFs in a transverse plane. Left, Schematic of a transverse section that includes field L overlaid with a map of STRFs. The orientation of the eight-electrode array is also shown. Right, In the expanded view of the map, each column corresponds to an electrode (numbered 1-8) and each row to a depth of recording. The spacing between neighboring electrodes was $\sim 200 \mu \mathrm{m}$, and recordings were made at eight depths in $200 \mu \mathrm{m}$ steps. The dotted line indicates borders between field L layers, and the empty squares are the sites that did not yield statistically significant STRFs. In this example map, the correlation coefficients between PSTH and prediction ranged from 0.29 (row 1, electrode 2) to 0.78 (row 3, electrode 4) with a mean of 0.57. Layer-dependent changes in temporal tuning and a mediolateral broadening in spectral tuning are evident. M, Medial; L, lateral.

representation, the stimulus was filtered into different frequency bands by a series of overlapping Gaussian filters whose center frequencies were logarithmically spaced between 250 and $10,112 \mathrm{~Hz}$. For each frequency band, the amplitude envelope was extracted using the analytic signal. The resulting representation of the stimulus as a series of log amplitude envelopes was used to compute the raw spike-triggered averages (STAs) (Gill et al., 2006). The raw STAs were computed at $1 \mathrm{~ms}$ resolution based on multiunit and single-unit spike times evoked by a continuously changing stimulus of 25 min duration. Multiunit spike times were obtained by thresholding the neural activity at three times the SD of a $5 \mathrm{~s}$ neural record. Single-unit spike times were obtained by sorting spikes from the raw waveform using a commercial spike sorter (Offline Sorter; Plexon). A clear separation of clusters of spike waveform projections in the two-dimensional principal component space and the requirement for a refractory period ( $<0.5 \%$ violation of a $1 \mathrm{~ms}$ refractory period) were used as criteria for single units. An STA was considered significant if at least 20 points of this STA were greater than six times the SD of the acausal part of the STA (i.e., the $100 \mathrm{~ms}$ window after a spike).

To correct for stimulus correlations, the raw STAs were divided by the stimulus autocorrelation in the frequency domain (Theunissen et al., 2000; Sen et al., 2001). During the decorrelation procedure, dividing by the autocorrelation may amplify noise, since higher modulation frequencies may be inadequately sampled. Therefore, we used a regularization procedure in which the eigenvalues of the stimulus autocorrelation matrix below a cutoff value were exponentially attenuated with a time constant of 100 eigenvalues. Typically, 30 different cutoff values were tested, which yielded a series of candidate decorrelated STAs, and the cutoff was set to maximize the Kullback-Leibler (KL) distance between the probability distributions $P(x \mid$ spike $)$ and $P(x)$ :

$$
D_{\mathrm{KL}}=\int d x P(x \mid \text { spike }) \log _{2}\left[\frac{P(x \mid \text { spike })}{P(x)}\right],
$$

where $P(x)$ is the probability distribution of all stimulus projections $x$ on a candidate decorrelated STA and $P(x \mid s p i k e)$ is the probability distribution of stimulus projections with only the parts of the stimulus that led to a spike (Sharpee et al., 2006). To avoid overfitting, we computed the raw
STA based on $80 \%$ of the data and then computed the KL distance by obtaining projection values using the remaining $20 \%$ of the stimulus that was not used to compute the STA. This procedure was repeated for five different $80 \% / 20 \%$ parts of the data. The mean of the five decorrelated STAs that maximized the KL distance is the STRF.

How well a STRF predicted the actual response of a neuron (the "prediction quality" of a STRF) was measured by calculating the correlation coefficient (CC) between the measured peristimulus time histogram (PSTH) for a repeated stimulus segment and the firing rate to that segment predicted by the STRF. To generate the predicted firing rate, first, the stimulus was convolved with the STRF. Then, a gain function given by $P(x \mid$ spike $)<r>/ P(x)(<\mathrm{r}>$, mean firing rate; Brenner et al., 2000) was applied to the convolution output. CCs were computed between the smoothed PSTH and the prediction. For each STRF, the size of a smoothing window (Hanning) was chosen to maximize the CC value (range, 16-128 ms) (Theunissen et al., 2000; Sen et al., 2001). STRFs that yielded $\mathrm{CC}$ values of $<0.2$ were excluded from analysis. In practice, this criterion eliminated only a small number of significant STAs from analysis (14 multiunits and 3 single units from an original population of 539 sites with significant STAs).

To examine whether temporally broad STRFs in the output layers of field L might have resulted from blurring of temporally narrow features by triggering on a series of successive spikes, we recomputed STRFs based only on the spikes preceded by a silent period longer than $60 \mathrm{~ms}$ (see the text for Fig. 5 in Results). We chose $60 \mathrm{~ms}$ because the interspike interval (ISI) distributions of our recording sites followed an exponential distribution for ISIs $>60 \mathrm{~ms}$, indicating that spikes are independent in this regime (i.e., Poisson regime) (Agüera y Arcas et al., 2003). In L1 and L3 combined, a total of 190 sites yielded significant STRFs, and 25 STRFs that clearly contained a mixture of slow and fast features were excluded from this analysis. Of these 165 STRFs based on isolated spikes, we compared 73 temporally broad STRFs ( $>25 \mathrm{~ms}$ ) against L2 STRFs based on all spikes.

STRF map analysis. To create a spatial map of STRFs, we mapped our recording sites onto brain sections based on the lesions. A series of lesions with a known separation $(500 \mu \mathrm{m})$ made at the end of a mapping experiment provided a depth reference so that recording sites could be mapped 
A

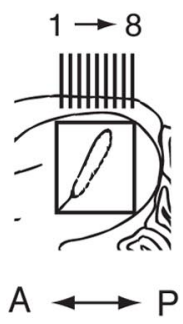

L1

L2

L3

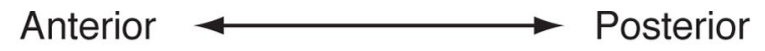

B

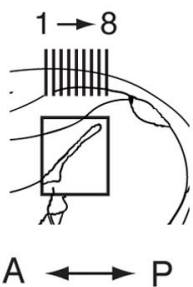

12

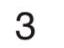

4

5

6

7

8

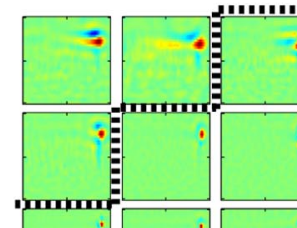

(1)

$+$
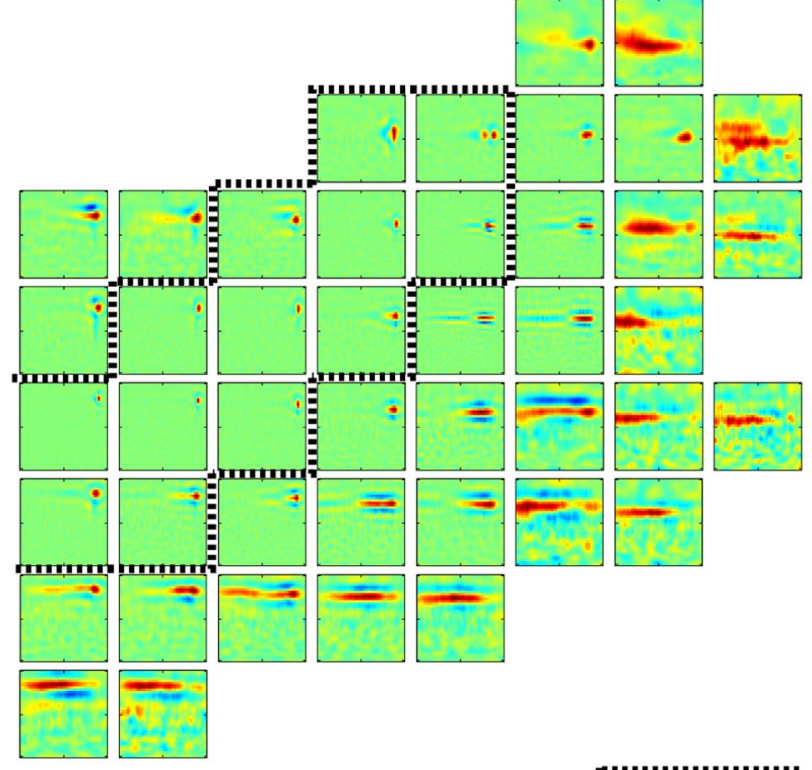

$=$
$=$
$=$
$\square$
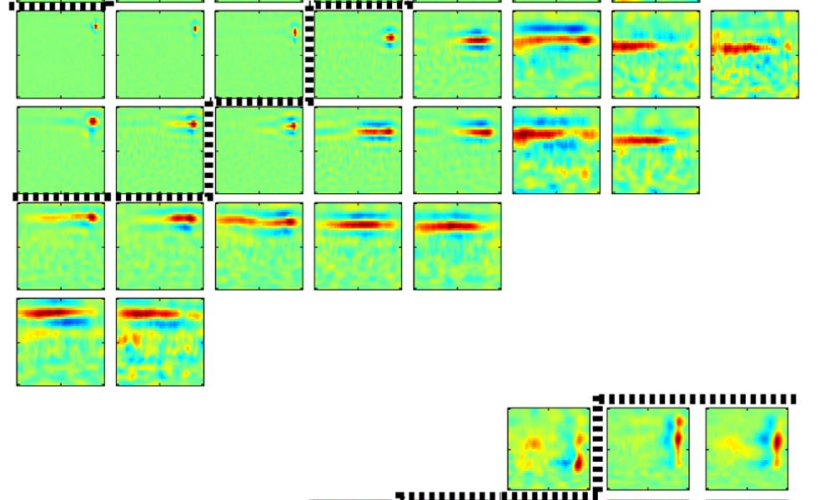

\section{L1}

.

L2

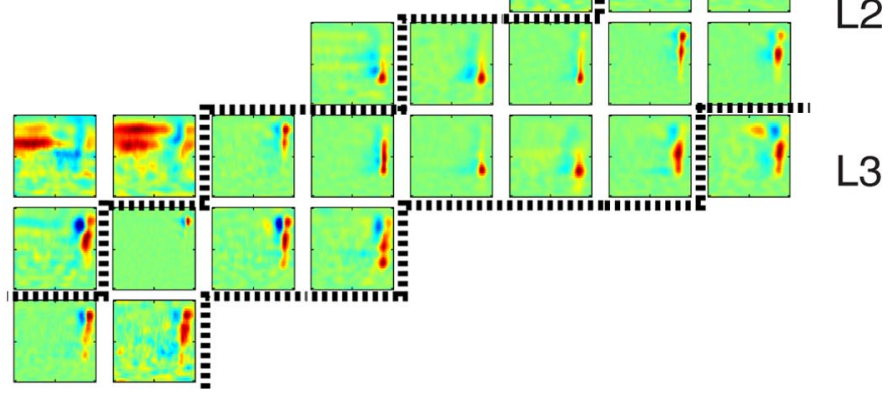

L2

Figure 4. Example maps of STRFs in two different parasagittal planes. $\boldsymbol{A}, \boldsymbol{B}$, Schematics of parasagittal sections at different distances from the midline $(\boldsymbol{A}, 0.7 \mathrm{~mm} ; \boldsymbol{B}, 1.8 \mathrm{~mm})$ with a drawing of the electrode arrays (left) and the corresponding maps of STRFs (right). Recordings were made in $250 \mu \mathrm{m}$ steps, and only statistically significant STRFs are shown. The dotted lines indicate borders between field $L$ layers. Note that layer-dependent changes in temporal tuning extend along the rostrocaudal dimension and that spectral tuning is narrower in the medial plane $(\boldsymbol{A})$ than in the lateral plane (B). $\boldsymbol{A}$, The mean of correlation coefficients between PSTH and prediction were 0.56 [range, 0.25 (row 5, electrode 7) to 0.74 (row 6, electrode 5)]. $\boldsymbol{B}$, The mean of correlation coefficients were 0.48 [range, 0.27 (row 3, electrode 1) to 0.71 (row 4, electrode 2)]. A, Anterior; $P$, posterior.

onto histological sections. Because we made lesions only at the first, fourth, and eighth columns, depths in the intervening columns were determined based on the closest column that had lesions. To assign recording sites to the layers of field L, the boundary of the L2 layer was determined based on the CB1 staining. Once the boundary of L2 was determined, the sites that fell between L2 and the mesopallial lamina (LaM) were categorized as L1, and all sites below L2 were categorized as L3. The sites above LaM were labeled as caudal mesopallium (CM) sites $(n=109)$ and were not included in the analysis. To summarize the mediolateral trend in tuning, the recording sites were divided into three groups (see Figs. 5-8): (1) sites from the parasagittal plane at $0.6 \mathrm{~mm}$ from the midline and the two most medial columns of the transverse plane; (2) sites from the parasagittal plane at $1.2 \mathrm{~mm}$ and the third and fourth columns of the transverse plane; (3) sites from the parasagittal plane at $1.8 \mathrm{~mm}$ and the remaining lateral columns of the transverse plane. The boundaries for the three mediolateral divisions corresponded to $\sim 0.9$ and $1.4 \mathrm{~mm}$ from the midline.

To create quantitative maps of spectrotemporal tuning, two parameters of field L STRFs were measured: spectral width and temporal width of the excitatory parts of the STRFs (see Figs. 5A, 6A). The spectral or temporal width was measured as the width of a spectral or temporal cross section (interpolated to $100 \times$ the original resolution) at $25 \%$ of the peak.
Because frequencies of STRFs are expressed on a logarithmic scale, the spectral width is expressed in octaves ( spectral width $=\log (\mathrm{f} 2 / \mathrm{f} 1) / \log (2)$, where $\mathrm{f} 1$ and $\mathrm{f} 2$ are two different frequencies). When more than one peak was present, the width with respect to the highest peak was used.

\section{Results}

To investigate the spatial organization of STRFs, we recorded neural activity of field L neurons in multiple anatomical planes. The field L complex of zebra finches consists of three layers (L1, L2, and L3), with the thalamic input layer L2 in the middle and the output layers L1 above and L3 below L2 (Fig. $1 A$ ). We first mapped STRFs of field L neurons in a transverse plane at the approximate rostrocaudal midpoint (206 sites in five birds; see Fig. 3). To further investigate the rostrocaudal organization of field L, we went on to map STRFs in three parasagittal planes (319 sites in nine birds; see Fig. 4).

To identify STRFs of field L neurons, we used a stimulus whose frequency bands contain independent amplitude modulations that reflect the statistics of zebra finch song (Fig. $1 B$; Nagel and Doupe, 2008). This stimulus has several advantages. First, 


\section{A}

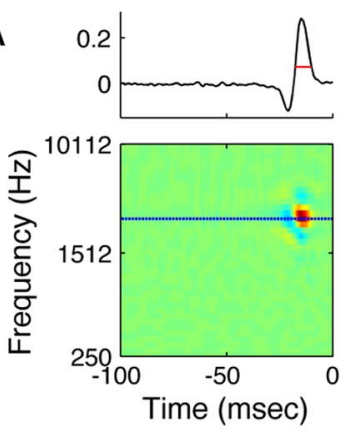

B

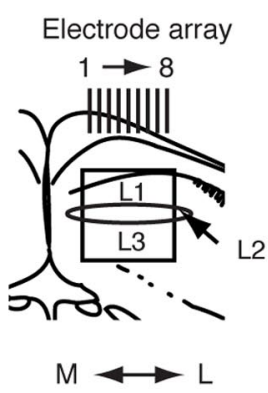

\section{Temporal width}

Medial Lateral

$\begin{array}{lllllllll}1 & 2 & 3 & 4 & 5 & 6 & 7 & 8 & \mathrm{msec}\end{array}$

L1

L2
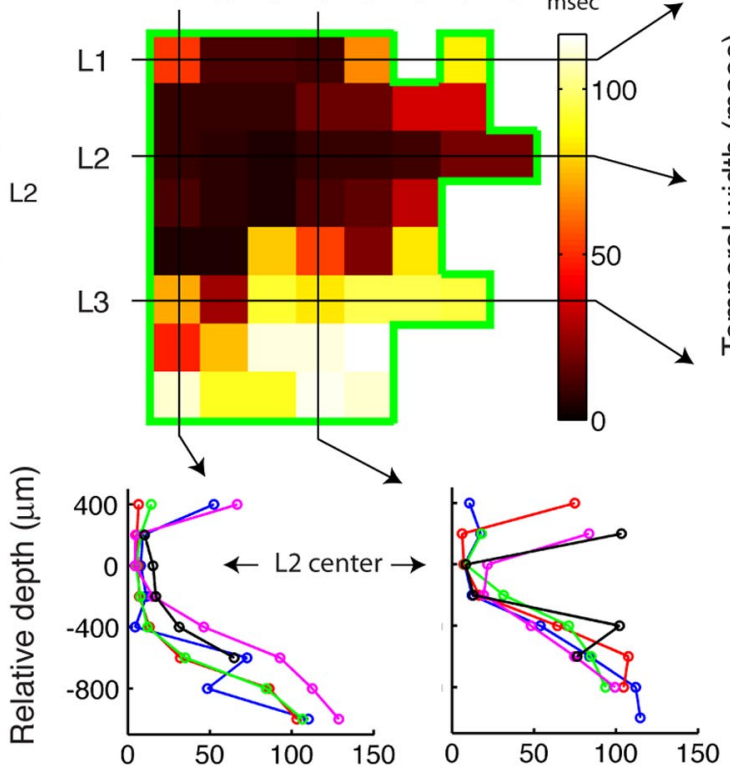

Temporal width (msec)

C

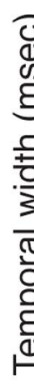

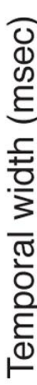

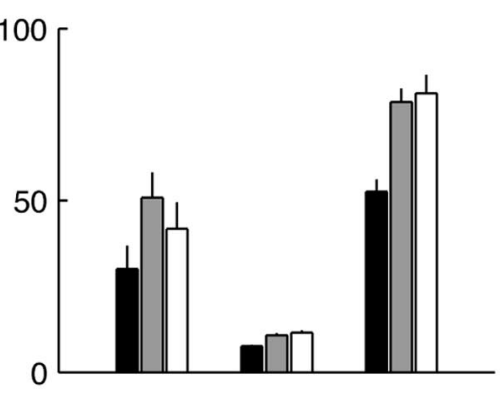

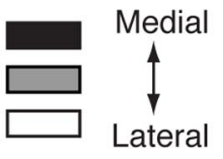
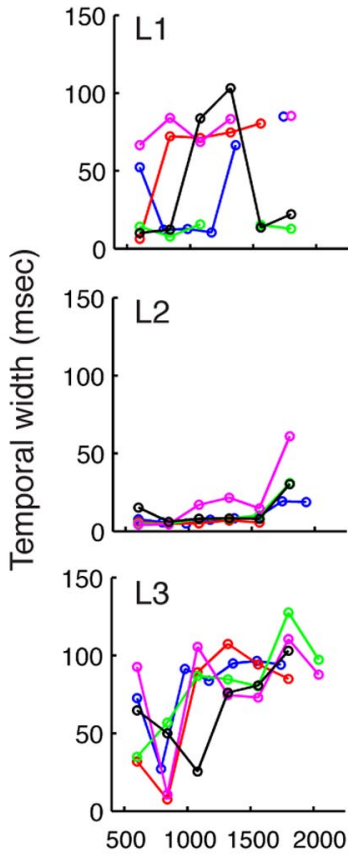

Distance $(\mu \mathrm{m})$

\section{L1 L2 L3}

Figure 5. Spatial organization of temporal tuning. $\boldsymbol{A}$, Temporal tuning width was measured at $25 \%$ of the peak of the temporal slice through the peak activity. $\boldsymbol{B}$, Spatial map of temporal width in a transverse plane. The example heat map (generated from the STRF map shown in Fig. 3) illustrates the spatial distribution of temporal widths. Each square corresponds to a STRF at a recording site (red, narrow temporal width; yellow, broad width). The sites outside the green boundary of the heat map did not yield significant STRFs. To illustrate the spatial distribution for different birds ( $n=5$ birds), plots of STRF temporal widths as a function of location are shown at a subset of columns and rows. The three plots on the right show temporal width as a function of the distance from the midline for each field $L$ layer. The two plots below the heat map show temporal width as a function of relative depth (zero at the center of L2), at two different columns (electrodes 1 and 4). In these plots, different line colors denote different birds (the blue is from the heat map shown). The patterns are generally consistent across birds. C, Summary of the spatial organization of temporal tuning for all sites. The mediolateral extent of field $\mathrm{L}$ is divided into three divisions for each layer (see Materials and Methods), and the mean for each of the nine subregions is plotted. In all three mediolateral subdivisions, mean temporal widths are significantly narrower in L2 than in L1 and $\mathrm{L} 3$ and narrower in $\mathrm{L} 1$ than in $\mathrm{L} 3$ ( $p<0.01$, ANOVA, Bonferroni correction). The most medial subdivisions (black bars) in L2 and L3, but not in L1, have significantly narrower temporal widths than the more lateral subdivisions (L1: $p=0.15$; L2 and L3: $p<0.01$, ANOVA, Bonferroni correction). Error bars denote SEM. 
because of its rich spectrotemporal structure, it is very effective at driving field L neurons. In fact, neural activity obtained at fixed depths without specifically searching for auditory units generally yielded clean STAs (Figs. 2-4). Second, the limited correlations between frequency bands in the stimulus yield STAs that are highly similar to the final decorrelated STRFs. Third, the amplitude modulation of the frequency bands has a Gaussian distribution, allowing the stimulus correlations to be mathematically removed from the STAs (Theunissen et al., 2000; Sharpee et al., 2006; see Materials and Methods).

\section{Distinct auditory responses across field $\mathrm{L}$ layers}

As an electrode array advanced through the layers of field L, distinct, sound-evoked patterns of neural activity emerged. Figure 2 depicts typical examples of neural activity evoked by a repeated segment of our stimulus in sites from L1, L2, and L3. In L2 (Fig. $2 B, C$ ), multiunit neural activity was precisely aligned at specific parts of the stimulus, as indicated by sharp peaks in PSTHs. The neural responses yielded STRFs that were narrowly tuned in time (Fig. $2 B, C$, right). The STRFs from sites in the lateral part of $\mathrm{L} 2$ tended to have broad spectral widths (Fig. 2C, right). In L1 and L3, spikes also reproducibly occurred at specific parts of the stimulus, but PSTHs had broader peaks, reflective of more sustained firing, responding to relatively slowly varying aspects of the stimulus (Fig. $2 A, D$ ). Neurons in these output layers thus tended to yield temporally broad STRFs, representing slowly varying sound features (Fig. $2 A, D$, right). The prediction quality of STRFs was assessed using the CC between the measured PSTHs (Fig. $2 A-D$, black lines) and firing rates to the same stimulus predicted using the STRFs (Fig. $2 A-D$, green lines). The full distribution of the CC values is shown in Figure $2 E$. Importantly, the mean $(\mathrm{SD}, 0.53 \pm 0.14)$ and range of these values were comparable to previous studies (Nagel and Doupe, 2008; Woolley et al., 2009). Moreover, all three types of STRFs broadly tuned in at least one dimension (Fig. $2 A, C, D$ ) predicted firing rate only slightly less well than STRFs that were narrowly tuned in both time and frequency (e.g., temporal width $<25 \mathrm{~ms}$ and spectral width $<1$ octave; Fig. $2 B$; CC, $0.50 \pm 0.13$ vs $0.6 \pm 0.14$ ).

\section{Spatial organization of temporal tuning}

When we mapped all significant STRFs onto a grid of anatomical locations based on recording depth and subsequent anatomical verification (Figs. $1 C, 3,4 ; n=525$ sites in 14 birds), two patterns of spatial organization emerged, one related to temporal tuning and one related to spectral tuning.

The temporal tuning pattern revealed a clear layer dependence: narrow temporal tuning ("fast") in the input layer L2 and broad tuning ("slow") in the output layers L1 and L3. The concentration of fast STRFs in L2 and the predominance of slow STRFs in L1 and L3 are visible in the transverse as well as the parasagittal planes shown (Figs. 3, 4).

To quantify the spatial organization of temporal tuning, we measured the full width of the temporal slice of each STRF at 25\% of the peak height (Fig. 5A) and plotted the widths as a function of anatomical location (Fig. $5 B, C$ ). In the example heat map of temporal widths in a transverse plane (Fig. $5 B$ ), the L2 layer contains darker squares (i.e., narrow tuning), whereas the L1 and L3 layers contain lighter squares (broad tuning) (Fig. 5B). We plotted tuning widths along either rows or columns to overlay data from different birds; a subset of these plots is shown beside the example heat map in Figure $5 B$. The row plots illustrate that STRFs are generally narrow in time across the full mediolateral extent of L2. In contrast, broad temporal widths are common in
Table 1. Summary of temporal tuning widths

\begin{tabular}{lcll}
\hline & Medial & & Lateral \\
\hline L1 & $30 \pm 7$ & $51 \pm 7$ & $42 \pm 8$ \\
L2 & $8 \pm 0.5$ & $11 \pm 0.7$ & $12 \pm 0.7$ \\
L3 & $53 \pm 4$ & $79 \pm 4$ & $81 \pm 5$ \\
\hline
\end{tabular}

Data are in milliseconds.

L1, although they are interspersed with some STRFs narrower in time. L3 had two characteristics: (1) L3 had generally broad widths, even more so than L1; and (2) there was a region of narrower widths in medial L3 (Fig. 5B, plots on the right). The plots for the first and fourth columns of the heat map confirm the clear difference in temporal widths across layers, with $\mathrm{L} 3$, in particular, showing a gradual increase in breadth of temporal tuning with distance from the input layer. This pattern was highly consistent across birds (Fig. 5B, bottom plots).

To summarize the results from all four mapping planes, we combined data from transverse and parasagittal planes by dividing the mediolateral extent of field $\mathrm{L}$ into three divisions (Fig. $5 \mathrm{C}$, Table 1; see Materials and Methods). In the bar graph (Fig. 5C), the striking pattern of temporal tuning that broadens from input to output layers is consistent for all field $\mathrm{L}$ mediolateral subdivisions $(p<0.01$, one way ANOVA for each mediolateral division followed by multiple comparisons with Bonferroni correction). Between the two output layers, temporal widths were significantly broader in L3 than in L1 (Fig. 5C). Within L2 and L3, more medial sites (Fig. 5C, black bars) had significantly tighter temporal tuning than more lateral sites (Fig. $5 C$, gray and white bars) in the same layer ( $p<0.01$, ANOVA, Bonferroni correction).

In the case of slow STRFs, a concern is that triggering on successive spikes during relatively sustained periods of firing (Fig. $2 A, D)$ could theoretically have resulted in temporally broad STRFs simply by blurring a temporally narrow feature. That is, for example, if the feature triggering a response was actually 10 $\mathrm{ms}$ long but always resulted in 10 spikes in short succession, the spike-triggered feature would appear to be much longer. To examine this issue, we recomputed STRFs based solely on spikes that occurred in isolation (interspike intervals $>60 \mathrm{~ms}$; see Materials and Methods) to investigate STRFs obtained in the absence of any potential blurring by closely spaced spikes. This is a very conservative measurement, which by examining only the first spike of any period of maintained firing likely greatly underestimates STRF temporal width for cells that respond to slow stimulus features. Nonetheless, after this recalculation, the temporal widths of temporally broad STRFs in L1 and L3 (those with original temporal widths $>25 \mathrm{~ms}$ ), although slightly decreased, remained clearly broader in time than those in L2 $[p<0.01 ; 35.0 \pm$ $1.4 \mathrm{~ms}(n=73)$ vs $9.8 \pm 0.4 \mathrm{~ms}(n=180)]$. The potential blurring by tonic firing could only be as much as the decrease in STRF width evident in this isolated spike calculation (35\% on average) if the putative brief sound feature abruptly ended after eliciting the first spike of a maintained firing period, whereas all the later spikes simply followed the first. This extreme scenario is unlikely, however, and the actual error attributable to blurring, if any, should be much smaller than the upper bound represented by considering only isolated spikes. Importantly, the significant difference between temporally broad STRFs in L1 and L3 and those in L2 remaining after this calculation demonstrates that even if temporal blurring were a contributing factor in temporally broad STRFs, it could not account for the observed difference in temporal tuning between input and output layers. 
A

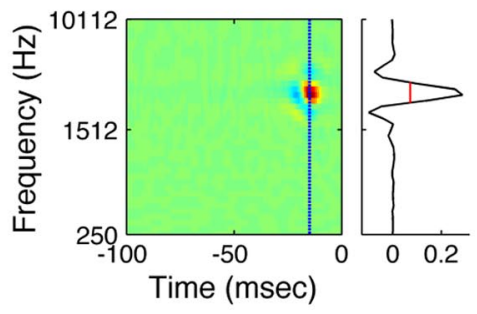

B

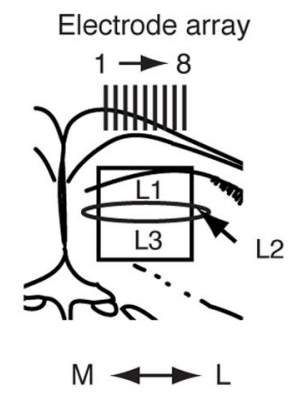

\section{Spectral width}
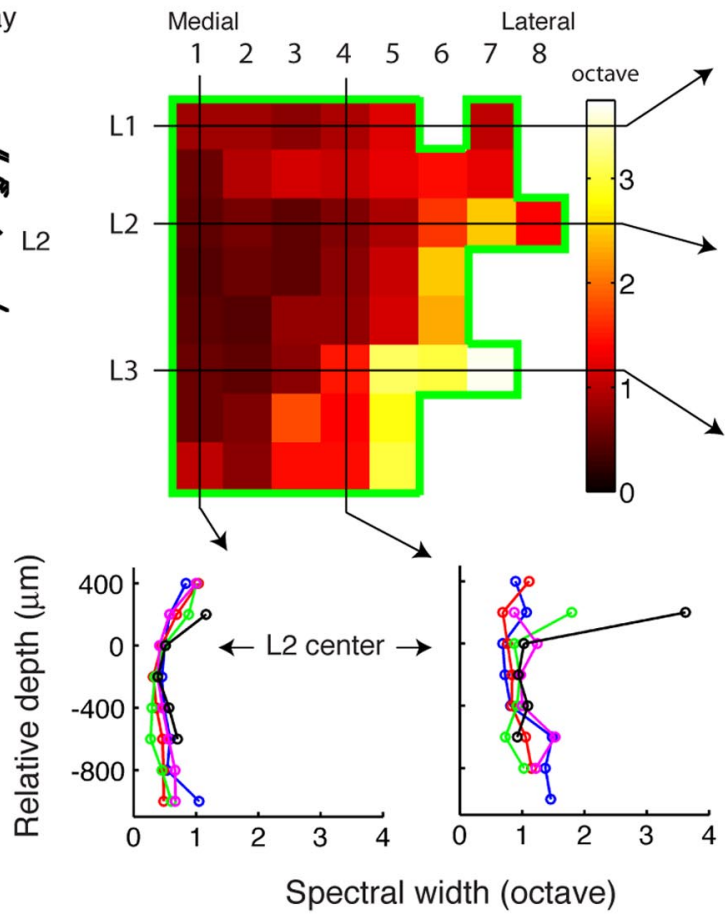

Spectral width (octave)

C

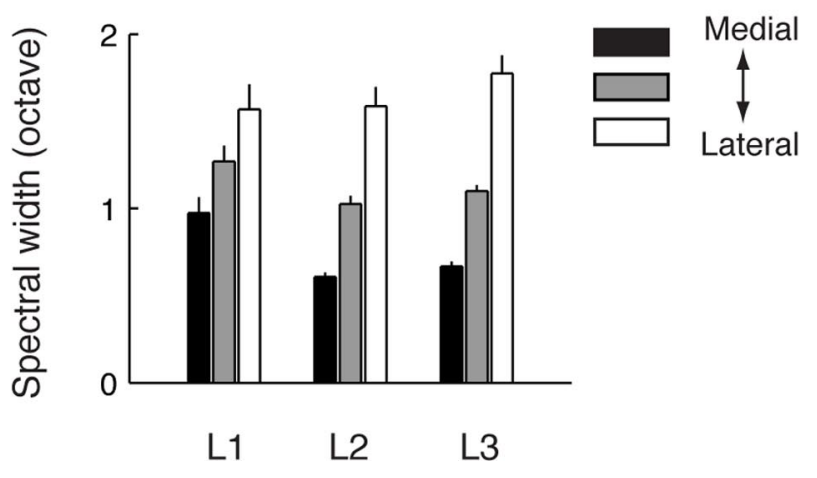

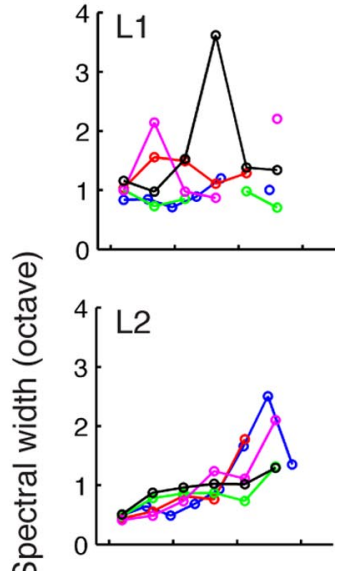

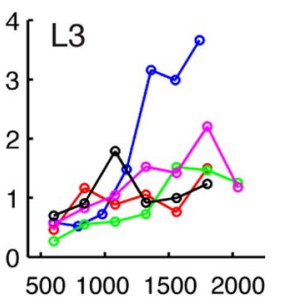

Distance $(\mu \mathrm{m})$

Figure 6. Spatial organization of spectral tuning. $A$, Spectral tuning width was measured at $25 \%$ of the peak of the spectral slice through the peak activity. $\boldsymbol{B}$, Spatial map of spectral width in a transverse plane. The same arrangement as in Figure 5, but showing spectral widths. The example heat map (generated from the STRF map shown in Fig. 3) illustrates the spatial distribution of spectral widths. The three plots on the right show STRF spectral width as a function of the distance from the midline for each field L layer. The two plots below the heat map show spectral width as a function of relative depth (zero at the center of L2), at two different columns (electrodes 1 and 4). Note the mediolateral broadening of spectral widths in L2 and L3. C, Summary of the spatial organization of spectral tuning for all sites. In all three layers, spectral widths broaden laterally ( $p<0.01$, ANOVA, Bonferroni correction). In the two more medial divisions (black and gray bars), L1 has significantly broader spectral tuning than L2 and L3 (black bars, $p<0.01$; gray bars, $p<0.051$, ANOVA, Bonferroni correction; white bars, $p=0.37$ ). Error bars denote SEM.

\section{Spatial organization of spectral tuning}

The second pattern of spatial organization evident in our data was a mediolateral gradient in spectral tuning. This is visible, for example, in the L2 sites of the transverse map (Fig. 3, third row) and in the progression of L2 STRFs from the most medial to the most lateral parasagittal plane (Fig. 4; see also Fig. $2 B$ ).

To quantify the spatial organization of spectral tuning, we measured the full width of the spectral slice of each STRF at 25\% 
Table 2. Summary of spectral tuning widths

\begin{tabular}{llll}
\hline & Medial & & Lateral \\
\hline L1 & $1.0 \pm 0.09$ & $1.3 \pm 0.09$ & $1.6 \pm 0.15$ \\
L2 & $0.6 \pm 0.03$ & $1.0 \pm 0.05$ & $1.6 \pm 0.11$ \\
L3 & $0.7 \pm 0.03$ & $1.1 \pm 0.04$ & $1.8 \pm 0.10$ \\
\hline
\end{tabular}

Data are in octaves.

of the peak height (Fig. $6 \mathrm{~A}$ ) and plotted the widths as a function of anatomical location (Fig. $6 B, C$ ). In the example heat map of spectral widths (Fig. 6B), medial sites contain darker squares (narrow tuning), whereas lateral sites contain lighter squares (broad tuning). The line plots for selected rows show that in L2 and L3, spectral widths broaden laterally (Fig. $6 \mathrm{~B}$, right). Along the first column, spectral widths narrow from L1 to L2 but then stay narrow from L2 to L3 (Fig. $6 \mathrm{~B}$, bottom left). Along the fourth column, spectral widths are broader than the first column and stay relatively constant across layers (Fig. $6 \mathrm{~B}$, bottom right).

We summarized the results from all four mapping planes as in the temporal tuning analysis above (Fig. 6C, Table 2). Mediolaterally, spectral tuning broadens in all three layers (Fig. $6 C$; $p<$ 0.01 for all three layers, ANOVA, Bonferroni correction). Across layers, no strong pattern exists except that medial L2 and L3 show narrower tuning than medial L1 (Fig. $6 C$, black and gray bars; $p<$ 0.05, ANOVA, Bonferroni correction).

In the mammalian auditory cortex and inferior colliculus, correlations between spectral width and best frequency (determined using pure tones) have been reported (Schreiner and Sutter, 1992; Imaizumi and Schreiner, 2007; Rodríguez et al., 2010). Although some individual maps showed such correlation (Fig. 3), overall this was not a consistent feature of our data, because spectral tuning remained broad laterally, regardless of whether best frequency was low (Fig. 3, electrodes 7 and 8) or high (Fig. $4 B$, electrodes $1-3,7$, and 8 ; correlations between best frequency and spectral width were insignificant in L1 and L2 and weak in L3 ( $r=$ $-0.26 ; p<0.01)$.

\section{Comparison between multiunits and single units}

Do the temporal and spectral patterns that we observed in multiunit recording exist at the single-neuron level? To begin to address this, we sorted single units from our multiunit sites and compared the resulting STRFs with those of the multiunit sites from which they had been sorted ( $n=35$; Fig. 7). A general concern with multiunit STRFs is that broad tuning could reflect the average tuning of a number of narrowly tuned neurons, each with different selectivity. This does not seem to be the case, however: we found that the recording sites with broadly tuned multiunit STRFs generally contained similarly broadly tuned single-unit STRFs (Fig. 7A, sites 1 and 2, Fig. $7 B$ ). In the case of temporal tuning, sites with temporally broad multiunit STRFs did occasionally yield single neurons that were substantially faster (Fig. 7A, site 3, Fig. 7B, bottom). This was rare (2 of 35), however. Moreover, neither in our single-unit nor in multiunit data did we observe temporally narrow STRFs with longer latencies, which would be one way to generate multiunit STRFs artificially broadened in time. Furthermore, in a number of sites (8 of 35), a broadly temporally tuned neuron emerged from a narrowly tuned multiunit site (Fig. $7 D$, site 4, Fig. $7 B$, bottom, upward lines). These data strongly suggest that temporally or spectrally broad STRFs are not simply an artifact of averaging the activity of differently tuned cells in multiunit recording. Furthermore, consistent with this, the layer dependence of temporal tuning (Fig. $7 C$, left) and the mediolateral broadening of spectral tuning (Fig.
$7 C$, right) are clearly evident in the population of single-unit STRFs (compare single-unit mapping with maps in Figs. $5 C$ and $6 C)$. We caution that our single-unit data set is relatively small, but the fact that the same spatial patterns as seen in more extensive multiunit data are already evident in our limited single-unit data further supports the idea that the spatial mapping of temporal and spectral tuning is a property of individual neurons.

We note that there is a substantial degree of local heterogeneity especially in temporal tuning (Fig. $7 D, E$ ). For this analysis, we compared the tuning of a single-unit site with that of the multiunit site remaining after subtracting the single-unit spike times ("nearby" multiunit site) ( $n=33$ sites). Spectral tuning width was generally well matched between a single-unit site and the nearby multiunit site (Fig. $7 D$, sites $1-4$, Fig. $7 E$, top). For temporal tuning, in $58 \%$ of the sites ( 19 of 33 ), temporal tuning width of a single-unit site was similar to that of the nearby multiunit site (Fig. 7D, sites 1 and 2, Fig. $7 E$, bottom), whereas in $42 \%$ of the sites (14 of 33), temporal tuning width differed by $>50 \%$ between a single unit and the nearby multiunit (Fig. $7 D$, sites 3 and 4, Fig. 7E). Although our single-unit data set is not extensive enough to allow accurate estimation of the degree of heterogeneity or of the proportions of different types of neurons in each subregion of field L, our results do show that nearby neurons in field L can have significantly different temporal tuning properties. Despite this local mixing of neighboring fast and slow neurons, our systematic multiunit and a smaller sample of singleunit recording detected a clear transition in temporal tuning from input to output layers. Therefore, the spatial pattern in temporal tuning most likely indicates a sharp increase in the proportion of neurons with broad temporal tuning in the output layers of field L.

\section{Spatial clustering of STRF types}

To examine whether spectral and temporal tuning are jointly represented in a spatially organized manner, we constructed a joint distribution of spectrotemporal tuning for all of our STRFs (Fig. $8 A ; n=525$ sites). Different regions of this joint distribution represent different types of spectrotemporal tuning, with neurons tightly tuned in both time and frequency at the origin, neurons tightly tuned in time but increasing in frequency breadth along the spectral $(y)$ axis, neurons sharply tuned in frequency but increasing in temporal width along the time $(x)$ axis, and cells broadly tuned in both dimensions lying off both axes. More than three quarters of STRFs had narrow tuning at least in one dimension (similar to Nagel and Doupe, 2008), although we also found a significant number of STRFs with broad tuning in both dimensions.

We then asked whether different subregions of field L represent specific regions of the joint distribution. As in Figure $5 C$ and $6 C$, each field $L$ layer was divided into three mediolateral divisions (Fig. $8 B-D$, top insets). In Figure $8 B-D$, the joint distribution for each layer, along with a pair of typical STRFs, is shown, with different colors denoting mediolateral divisions. Conspicuously, the STRFs from the input layer L2 lie strongly clustered along the spectral axis, indicating narrow temporal tuning (Fig. $8 B$, scatter plot with histograms of temporal tuning above). Also notable is the gradual broadening of spectral tuning, which is visible in the joint distributions and even more clearly in the spectral histograms to the right of these distributions (Fig. $8 \mathrm{~B}$ ). In particular, the STRFs from medial L2 occupy the left bottom corner (Fig. 8 B, red dots), indicating narrow tuning in both time and frequency. In the output layer L3, the majority of STRFs lie along the temporal axis, especially for medial L3, indicating nar- 
row spectral tuning with varying degrees of temporal tuning (Fig. 8C); as in L2, there is gradual spectral broadening laterally (Fig. $8 C$, green and blue dots), resulting in broadband neurons in the lateral regions of L3. Finally, the other output layer, L1, contains intermingled L2-like STRFs and STRFs with broad tuning in time and frequency (Fig. 8D), although, again, the most medial region is dominated by neurons relatively narrower in frequency and time. These results illustrate that STRFs in different subregions of field L cluster in terms of their spectrotemporal tuning.

\section{Discussion}

By systematically mapping STRFs, we found an orderly representation of sound features in finch field L (Fig. 9A), with separate orthogonal axes mapping spectral and temporal width. First, temporal tuning differed remarkably across field L layers, with fast cells concentrated in the input layer L2 and slow cells emerging in the output layers L1 and L3. Second, in all layers, spectral tuning broadened mediolaterally. Finally, spectrotemporal tuning types could be mapped onto different subregions of field L (Fig. 9B). Our results indicate a strikingly spatially organized hierarchical processing of sound features at the first stages of the songbird auditory telencephalon.

\section{Multielectrode mapping of STRFs}

To investigate the spatial organization of spectrotemporal modulations in field L, which has not been evident in most prior studies, we took a systematic mapping approach, sampling multiunit and singleunit neural responses from an array of recording sites at regular intervals. This approach allowed us to obtain an overall spatial organization within each animal.

The spatial patterns we observed are most likely the result of a preponderance of different types of STRFs in different subregions of field L. This conclusion is supported by the fact that we observed essentially the same temporal and spectral patterns in our relatively limited sample of single-unit as in multiunit STRFs (Fig. $7 C)$. We note that our maps are not incompatible with some local heterogeneity, especially in temporal tuning. This was not evident in L2, where despite its small densely packed neurons (Fortune and Margoliash, 1992), we observed extremely tight temporal tuning in multiunit STRFs, suggesting a high degree of temporal homogeneity. In our limited comparison of single and multiple units in
A

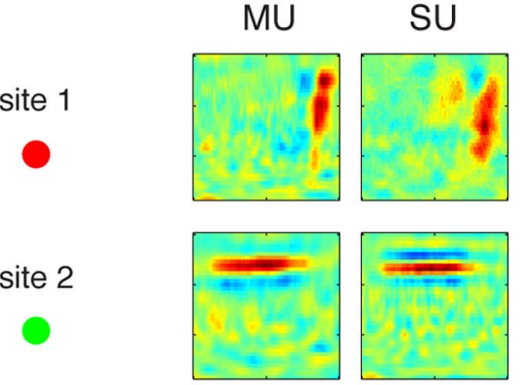

site 3

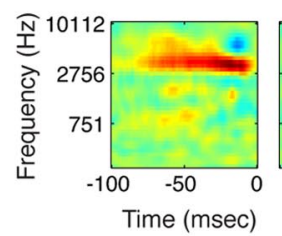

C

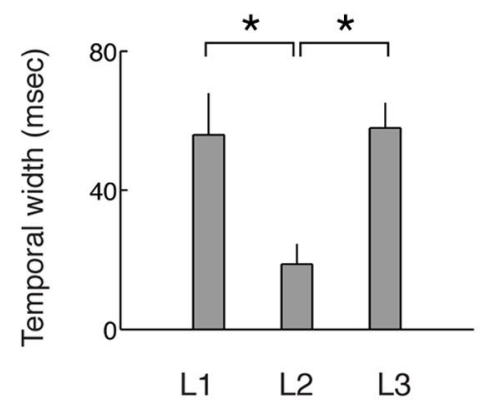

D

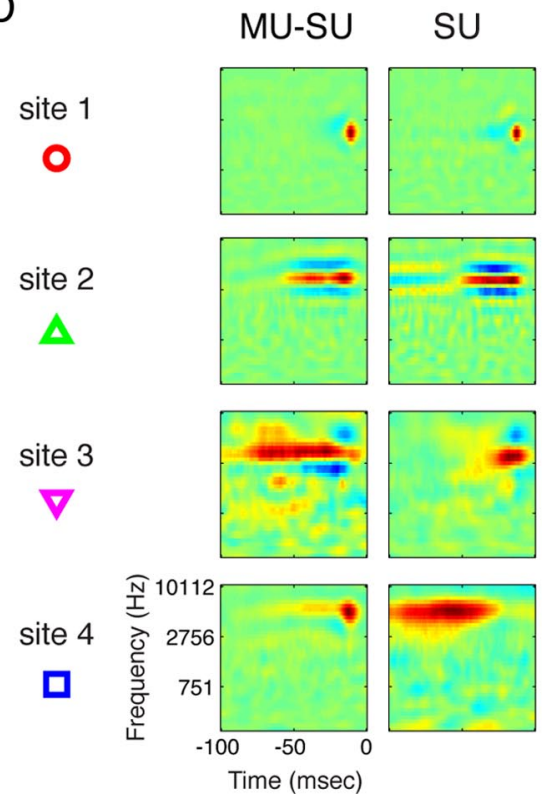

B
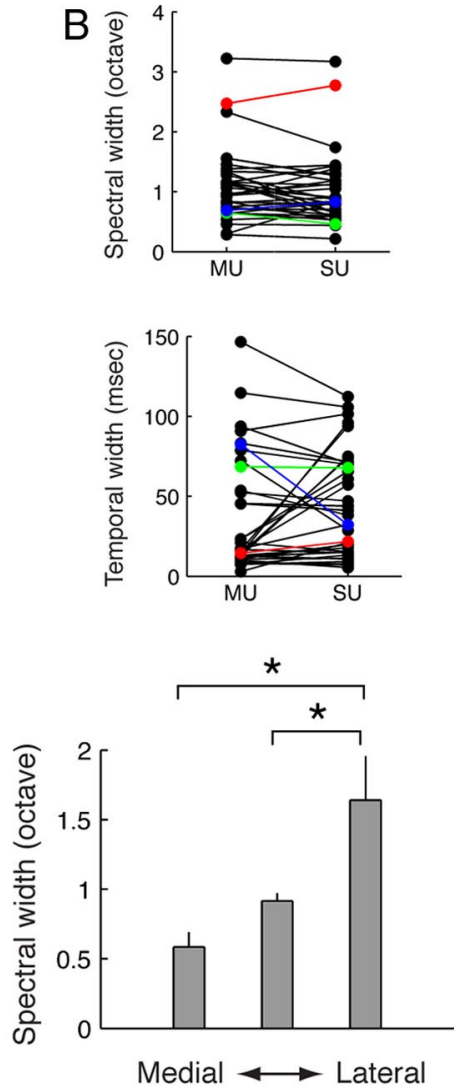

E
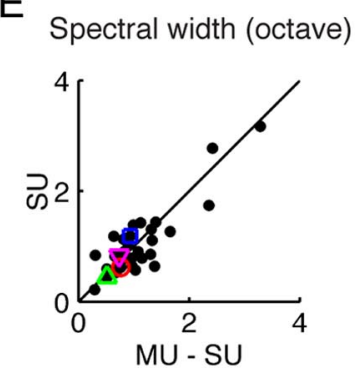

Temporal width (msec)

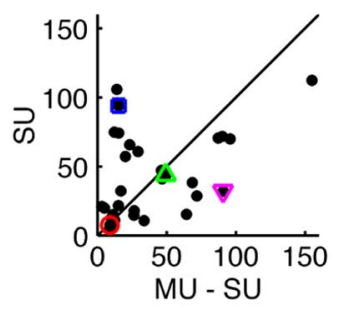

Figure 7. Comparison between multiunits and single units. $A$, Three example sites where both multiunit and single-unit STRFs were obtained. Each row shows a multiunit STRF (MU; left) and a single-unit STRF from the same site (SU; right). In sites 1 and 2, both temporal and spectral tuning widths are in good agreement. Site 3 shows a site where spectral tuning was in agreement between MU and SU, but temporal tuning differed, providing evidence for a mixture of fast and slow neurons in the MU. Symbols with different colors indicate corresponding data points in $\boldsymbol{B}$. $\boldsymbol{B}$, Population data for pairwise comparisons of spectral width (top) and temporal width (bottom) between MU and SU STRFs $(n=35)$. Colored symbols indicate example STRFs shown in $A$. C, Spatial organization of temporal (left) and spectral (right) width of SU STRFs. * Statistical significance ( $p<0.05$, ANOVA with Bonferroni test). $\boldsymbol{D}$, Comparison between MU STRFs based on spikes remaining after subtracting SU spikes (MU-SU; left column) and SU STRFs (right column) from the same site. Sites 1 and 2 show sites where the two STRFs are in good agreement; in contrast, for sites 3 and 4, the two STRFs are well matched for spectral width but differ in temporal width, indicating that nearby neurons can have substantially different temporal tuning. Colored symbols indicate corresponding data points in E. E, Population data comparing spectral (top) and temporal (bottom) width between MU-SU STRFs and SU STRFs $(n=33)$. 
A

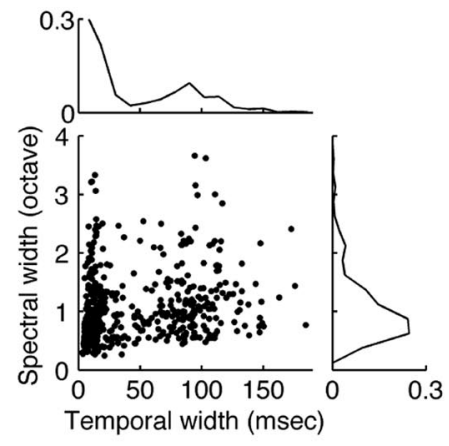

L3
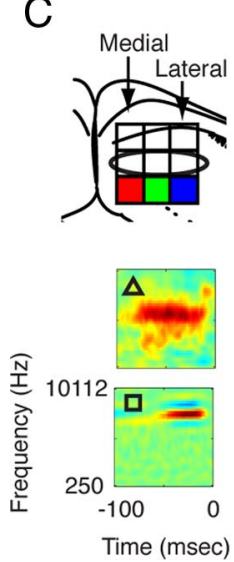

B
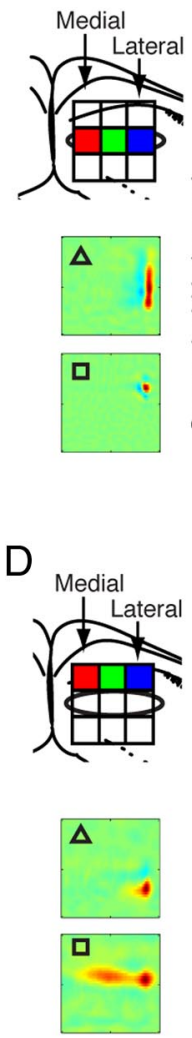

L2

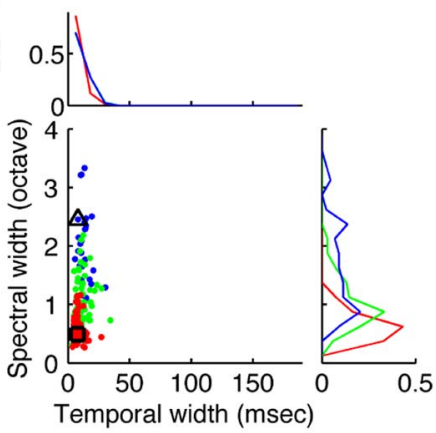

L1
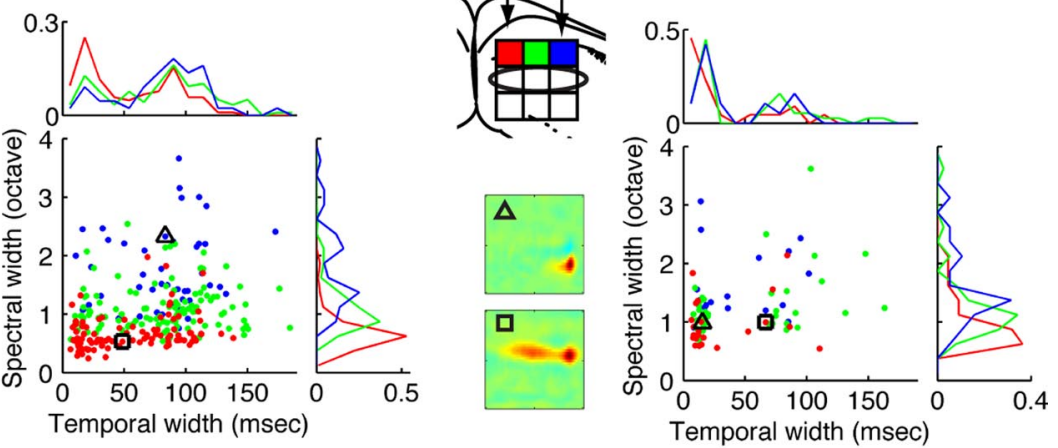

Figure 8. Joint distribution of spectrotemporal tuning and its relationship with anatomical subregions in field L. A, Joint distribution of spectral and temporal tuning widths for all sites. Each dot corresponds to a recording site $(n=525)$. The histograms along the edges show the distribution of temporal widths (top) or spectral widths (right). $\boldsymbol{B}$ - $\boldsymbol{D}$, Joint distributions for individual subregions $(\boldsymbol{B}, \mathrm{L} 2 ; \boldsymbol{C}, \mathbf{L} 3 ; \boldsymbol{D}, \mathbf{L} 1)$. In the scatter plots and histograms, the mediolateral divisions are denoted with three colors (red, green, and blue). For each field L layer, two typical example STRFs are shown, and their location in the joint plot is indicated by a black square and a triangle. $\boldsymbol{B}$, In L2, all sites are lined up along the spectral axis, indicating tight temporal tuning. Note the mediolateral shift in spectral tuning both in the scatter plot and the histograms. C, In L3, most of the sites lie along the temporal axis, indicating narrow spectral tuning with a broad range of temporal tuning. Note the mediolateral shift in spectral tuning in the scatter plot and the histograms. $D, I n L 1$, a mixture of L2-like and L3-like tuning was found. The smaller number of sites in L1 reflects its relatively narrow anatomical extent.

L1 and L3 (Fig. 7D,E), however, about half of the sites contained both slow and fast neurons. Despite this, multiunit STRFs in these areas were markedly slow. Together, these results indicate that the layer-dependent broadening of temporal tuning seen here represents a sharp increase in the proportion of slow neurons from the input to the output layers.

\section{Spatially organized spectrotemporal tuning}

Previous studies have suggested differential temporal tuning across field L layers in birds. In the chick, latencies of tone-evoked responses, which may correlate with differences in temporal tuning (Langner et al., 1987), were shorter in L2 than in L1 and L3 (Heil and Scheich, 1991). In mynah birds, preferred amplitudemodulation frequencies differed across field L layers (Hose et al., 1987). In zebra finches, reverse correlation studies suggested that neurons in L2 were tuned to fast temporal modulations (Sen et al., 2001; Nagel and Doupe, 2008). Our data extend these findings by mapping field $\mathrm{L}$ in multiple anatomical planes using complex sounds, demonstrating a striking transition in temporal tuning between input and output layers of field L (Fig. 9A, left, schematic).

Neural mechanisms that determine temporal tuning are not well understood. Intrinsic firing properties of the neurons are likely to limit the fastest temporal modulation a neuron can fol- low and also to contribute to tuning (Joris et al., 2004; Nagel and Doupe, 2008). Synaptic mechanisms such as synaptic depression may also influence temporal tuning (Chance et al., 1998; Eggermont, 2002).

We found an organization in spectral width tuning that has not been reported previously in songbirds (Fig. 9A, right). The spectral tuning gradient suggests parallel channels with different degrees of spectral integration (Schreiner et al., 2000). We observed broadening of spectral tuning in all field L layers, even though these layers show markedly different temporal tuning, suggesting that spectral and temporal tuning arise from distinct mechanisms. Highly broadband neurons have been observed in both the songbird auditory midbrain (Woolley et al., 2006, 2009) and the thalamus (Amin et al., 2010). Therefore, there may be a broadband pathway that arises early in the avian auditory brainstem. It will be important to determine how the parallel spectral streams of field L propagate into the higher auditory stations CM and caudomedial nidopallium (NCM), where higher-order response selectivities for auditory objects have been found (Gentner and Margoliash, 2003; Gill et al., 2008; Meliza et al., 2010; Jeanne et al., 2011).

It remains to be determined whether the same organization is observed when field L neurons are probed with fully natural stimuli such as song. In songbird midbrain, most neurons showed 
broader spectral tuning width when probed with song than with a noise stimulus (Woolley et al., 2006). Accordingly, the field L spectral gradient might also change depending on stimulus statistics. With respect to temporal tuning, it seems unlikely that the clear layer difference would disappear even with song stimuli. Probing with natural stimuli could reveal important quantitative differences in tuning, but we predict that the basic spectral and temporal patterns would be preserved.

When temporal and spectral tuning were jointly considered, different subregions of field $\mathrm{L}$ occupied limited regions of the spectrotemporal tuning space (Fig. 8 ). A converse way to look at this is illustrated in Figure 9B. When we divided up our STRFs into four different types based on their spectrotemporal tuning, we saw an anatomical clustering of STRF types: (1) STRFs narrow in both time and frequency in medial L2 (Fig. 9B, green dots); (2) STRFs narrow in time but broad in frequency in lateral L2 (Fig. 9B, blue dots); (3) STRFs broad in time but narrow in frequency in medial L3 (Fig. 9B, red dots); and (4) STRFs broad in both time and frequency primarily in lateral L3 (Fig. 9B, black dots). This remarkable anatomical clustering of different STRF shapes contrasts with results to date from the mammalian auditory cortex (see below) and may enable us to understand how cortical STRFs arise through the neural circuitry.

The diversity of forebrain STRFs has been proposed to underlie perceptual qualities of complex sounds (Woolley et al., 2009): filtering of sound by broadband fast STRFs could represent rhythm, narrowband slow STRFs could represent pitch, and STRFs broad in both dimensions could represent timbre. Our data suggest that such forebrain filtering of sounds for basic perceptual qualities occurs in a spatially organized and segregated manner, with separate axes focused on spectral and temporal width, respectively. As in visual cortex, higher-level auditory processing has also been proposed to be segregated into pathways specialized for different aspects of auditory objects (Tian et al., 2001; Lomber and Malhotra, 2008; Kusmierek and Rauschecker, 2009; Recanzone and Cohen, 2010). The separate axes we observed here in a primary auditory area might provide outputs relevant to such higher-level segregation.

\section{Comparison with the mammalian auditory system}

The strong layer dependence in temporal tuning contrasts with A1 data. Significant transformations in temporal tuning occur from inferior colliculus (IC) to auditory thalamus and then to A1 (Creutzfeldt et al., 1980; Miller et al., 2002; Joris et al., 2004; Ter-Mikaelian et al., 2007; Wang et al., 2008). Within A1, however, systematic recordings across cortical layers of A1 did not find a clear layer dependence in temporal tuning (Atencio and Schreiner, 2010a,b). Given the anatomical correspondence between $\mathrm{A} 1$ and field $\mathrm{L}$ as the first postthalamic station, this differ- ence suggests that field L subserves the same level of processing but that the layers of field L are organized in a more hierarchical manner than A1.

Using pure tones, multiple anatomical organizations for spectral tuning have been found in A1. Within each isofrequency lamina, spectral tuning width varies systematically (Schreiner et al., 2000). In addition, spectral tuning is narrower in the midfrequency portion of A1 (Imaizumi and Schreiner, 2007). The mediolateral spectral width gradient in field $\mathrm{L}$ does not seem to be a gradient within an isofrequency lamina since best frequencies can change along the same axis. Nor does it necessarily correlate with certain best frequencies. Therefore, unlike A1, the spectral gradient in field L seems to be mapped independently of tonotopy. It has been hypothesized that having a range of bandwidths enhances complex sound processing and that different spectral tuning underlies different percepts (Schreiner et al., 2000; Chi et al., 2005). Despite organizational differences, the field L spectral gradient likely represents parallel streams of spectral channels that serve similar functions to those hypothesized for the spectral gradients in A1.

Our data show an orderly map of STRFs, reminiscent of the systematic organization of mammalian visual cortex but not seen to date in A1. Studies of A1 show a wide diversity of STRF shapes, without clear spatial organization (Atencio and Schreiner, 2010a), although systematic mapping of STRFs across the A1 
surface remains to be done. Recent studies have, however, found orderly representations of spectrotemporal modulations in mammalian IC (Rodríguez et al., 2010; Baumann et al., 2011). The striking postthalamic organization seen here, combined with relatively fewer STRF categories (Nagel and Doupe, 2008; Woolley et al., 2009), suggests that the avian telencephalon may be governed by simpler and more systematic organizing principles than mammalian auditory cortex. Alternatively, basic organizational rules for auditory cortex could be shared across birds and mammals but could simply be more evident in songbirds.

The robust organization of field L may be an evolutionary adaptation of avian forebrain. An intriguing possibility is that this functional organization is more pronounced in the auditory forebrain of vocal learners, whose behavior critically depends on vocal communication and learning. Our results identify basic steps of complex sound processing in the auditory telencephalon in a species with well defined behavioral challenges. The exact organization may differ from the mammalian auditory cortex, but the sequence of processing stages is likely to have general implications for hierarchical processing of complex sounds.

\section{References}

Agüera y Arcas B, Fairhall AL, Bialek W (2003) Computation in a single neuron: Hodgkin and Huxley revisited. Neural Comput 15:1715-1749.

Amin N, Gill P, Theunissen FE (2010) Role of the zebra finch auditory thalamus in generating complex representations for natural sounds. J. Neurophysiol 104:784-798.

Atencio CA, Schreiner CE (2010a) Laminar diversity of dynamic sound processing in cat primary auditory cortex. J Neurophysiol 103:192-205.

Atencio CA, Schreiner CE (2010b) Columnar connectivity and laminar processing in cat primary auditory cortex. PLoS One 5:e9521.

Atencio CA, Sharpee TO, Schreiner CE (2009) Hierarchical computation in the canonical auditory cortical circuit. Proc Natl Acad Sci U S A 106:21894-21899.

Baumann S, Griffiths TD, Sun L, Petkov CI, Thiele A, Rees A (2011) Orthogonal representation of sound dimensions in the primate midbrain. Nat Neurosci 14:423-425.

Brenner N, Bialek W, de Ruyter van Steveninck R (2000) Adaptive rescaling maximizes information transmission. Neuron 26:695-701.

Chance FS, Nelson SB, Abbott LF (1998) Synaptic depression and the temporal response characteristics of V1 cells. J Neurosci 18:4785-4799.

Chi T, Ru P, Shamma SA (2005) Multiresolution spectrotemporal analysis of complex sounds. J Acoust Soc Am 118:887-906.

Creutzfeldt O, Hellweg F-C, Schreiner C (1980) Thalamocortical transformation of responses to complex auditory stimuli. Exp Brain Res 39:87-104.

Depireux DA, Simon JZ, Klein DJ, Shamma SA (2001) Spectro-temporal response field characterization with dynamic ripples in ferret primary auditory cortex. J Neurophysiol 85:1220-1234.

Dugas-Ford J, Ragsdale CW (2009) Markers of mammalian layer 5 and 6 cells demonstrate the subnuclear architecture of the avian arcopallium. Soc Neurosci Abstr 35:83.12.

Eggermont JJ (2002) Temporal modulation transfer functions in cat primary auditory cortex: separating stimulus effects from neural mechanisms. J Neurophysiol 87:305-321.

Eggermont JJ, Johannesma PM, Aertsen AM (1983) Reverse-correlation methods in auditory research. Q Rev Biophys 16:341-414.

Ferster D, Chung S, Wheat H (1996) Orientation selectivity of thalamic input to simple cells of cat visual cortex. Nature 380:249-252.

Fortune ES, Margoliash D (1992) Cytoarchitectonic organization and morphology of cells of the field L complex in male zebra finches (Taenopygia guttata). J Comp Neurol 325:388-404.

Gehr DD, Capsius B, Gräbner P, Gahr M, Leppelsack HJ (1999) Functional organisation of the field-L-complex of adult male zebra finches. Neuroreport 10:375-380.

Gentner TQ, Margoliash D (2003) Neuronal populations and single cells representing learned auditory objects. Nature 424:669-674.

Gill P, Zhang J, Woolley SMN, Fremouw T, Theunissen FE (2006) Sound representation methods for spectro-temporal receptive field estimation. J Comput Neurosci 21:5-20.
Gill P, Woolley SMN, Fremouw T, Theunissen FE (2008) What's that sound? Auditory area CLM encodes stimulus surprise, not intensity or intensity changes. J Neurophysiol 99:2809-2820.

Heil P, Scheich H (1985) Quantitative analysis and two-dimensional reconstruction of the tonotopic organization of the auditory field $\mathrm{L}$ in the chick from 2-deoxyglucose data. Exp Brain Res 58:532-543.

Heil P, Scheich H (1991) Functional organization of the avian auditory cortex analogue. II. Topographic distribution of latency. Brain Res 539:121-125.

Hose B, Langner G, Scheich H (1987) Topographic representation of periodicities in the forebrain of the mynah bird: one map for pitch and rhythm? Brain Res 422:367-373.

Hubel DH, Wiesel TN (1962) Receptive fields, binocular interaction and functional architecture in the cat's visual cortex. J Physiol 160:106-154.

Imaizumi K, Schreiner CE (2007) Spatial interaction between spectral integration and frequency gradient in primary auditory cortex. J Neurophysiol 98:2933-2942.

Jeanne JM, Thompson JV, Sharpee TO, Gentner TQ (2011) Emergence of learned categorical representations within an auditory forebrain circuit. J Neurosci 31:2595-2606.

Joris PX, Schreiner CE, Rees A (2004) Neural processing of amplitudemodulated sounds. Physiol Rev 84:541-577.

Karten HJ (1968) The ascending auditory pathway in the pigeon (Columba livia). II. Telencephalic projections of the nucleus ovoidalis thalami. Brain Res 11:134-153.

King AJ, Nelken I (2009) Unraveling the principles of auditory cortical processing: can we learn from the visual system? Nat Neurosci 12:698-701.

Kowalski N, Depireux DA, Shamma SA (1996a) Analysis of dynamic spectra in ferret primary auditory cortex. I. Characteristics of single-unit responses to moving ripple spectra. J Neurophysiol 76:3503-3523.

Kowalski N, Depireux DA, Shamma SA (1996b) Analysis of dynamic spectra in ferret primary auditory cortex. II. Prediction of unit responses to arbitrary dynamic spectra. J Neurophysiol 76:3524-3534.

Kusmierek P, Rauschecker JP (2009) Functional specialization of medial auditory belt cortex in the alert rhesus monkey. J Neurophysiol 102:1606-1622.

Langner G, Schreiner C, Merzenich MM (1987) Covariation of latency and temporal resolution in the inferior colliculus of the cat. Hear Res 31:197-201.

Lomber SG, Malhotra S (2008) Double dissociation of "what" and "where" processing in auditory cortex. Nat Neurosci 11:609-616.

Meliza CD, Chi Z, Margoliash D (2010) Representations of conspecific song by starling secondary forebrain auditory neurons: toward a hierarchical framework. J Neurophysiol 103:1195-1208.

Miller LM, Escabí MA, Read HL, Schreiner CE (2002) Spectrotemporal receptive fields in the lemniscal auditory thalamus and cortex. J Neurophysiol 87:516-527.

Müller CM, Leppelsack HJ (1985) Feature extraction and tonotopic organization in the avian auditory forebrain. Exp Brain Res 59:587-599.

Nagel KI, Doupe AJ (2008) Organizing principles of spectro-temporal encoding in the avian primary auditory area field L. Neuron 58:938-955.

Recanzone GH, Cohen YE (2010) Serial and parallel processing in the primate auditory cortex revisited. Behav Brain Res 206:1-7.

Rodríguez FA, Read HL, Escabí MA (2010) Spectral and temporal modulation tradeoff in the inferior colliculus. J Neurophysiol 103:887-903.

Scheich H, Bonke BA, Bonke D, Langner G (1979) Functional organization of some auditory nuclei in the guinea fowl demonstrated by the 2-deoxyglucose technique. Cell Tissue Res 204:17-27.

Schreiner CE, Sutter ML (1992) Topography of excitatory bandwidth in cat primary auditory cortex: single-neuron versus multiple-neuron recordings. J Neurophysiol 68:1487-1502.

Schreiner CE, Winer JA (2007) Auditory cortex mapmaking: principles, projections, and plasticity. Neuron 56:356-365.

Schreiner CE, Read HL, Sutter ML (2000) Modular organization of frequency integration in primary auditory cortex. Annu Rev Neurosci 23:501-529.

Sen K, Theunissen FE, Doupe AJ (2001) Feature analysis of natural sounds in the songbird auditory forebrain. J Neurophysiol 86:1445-1458.

Sharpee TO, Sugihara H, Kurgansky AV, Rebrik SP, Stryker MP, Miller KD (2006) Adaptive filtering enhances information transmission in visual cortex. Nature 439:936-942.

Singh NC, Theunissen FE (2003) Modulation spectra of natural sounds and 
ethological theories of auditory processing. J Acoust Soc Am 114:3394-3411.

Soderstrom K, Tian Q, Valenti M, Di Marzo V (2004) Endocannabinoids link feeding state and auditory perception-related gene expression. J Neurosci 24:10013-10021.

Terleph TA, Mello CV, Vicario DS (2006) Auditory topography and temporal response dynamics of canary caudal telencephalon. J Neurobiol 66:281-292.

Ter-Mikaelian M, Sanes DH, Semple MN (2007) Transformation of temporal properties between auditory midbrain and cortex in the awake Mongolian gerbil. J Neurosci 27:6091-6102.

Theunissen FE, Doupe AJ (1998) Temporal and spectral sensitivity of complex auditory neurons in the nucleus $\mathrm{HVc}$ of male zebra finches. J Neurosci 18:3786-3802.

Theunissen FE, Sen K, Doupe AJ (2000) Spectral-temporal receptive fields of nonlinear auditory neurons obtained using natural sounds. J Neurosci 20:2315-2331.

Tian B, Reser D, Durham A, Kustov A, Rauschecker JP (2001) Functional specialization in rhesus monkey auditory cortex. Science 292:290-293.
Vates GE, Broome BM, Mello CV, Nottebohm F (1996) Auditory pathways of caudal telencephalon and their relation to the song system of adult male zebra finches. J Comp Neurol 366:613-642.

Wang X, Lu T, Bendor D, Bartlett E (2008) Neural coding of temporal information in auditory thalamus and cortex. Neuroscience 157:484494.

Wang Y, Brzozowska-Prechtl A, Karten HJ (2010) Laminar and columnar auditory cortex in avian brain. Proc Natl Acad Sci U S A 107: $12676-12681$.

Wild JM, Karten HJ, Frost BJ (1993) Connections of the auditory forebrain in the pigeon (Columba livia). J Comp Neurol 337:32-62.

Woolley SMN, Fremouw TE, Hsu A, Theunissen FE (2005) Tuning for spectro-temporal modulations as a mechanism for auditory discrimination of natural sounds. Nat Neurosci 8:1371-1379.

Woolley SMN, Gill PR, Theunissen FE (2006) Stimulus-dependent auditory tuning results in synchronous population coding of vocalizations in the songbird midbrain. J Neurosci 26:2499-2512.

Woolley SMN, Gill PR, Fremouw T, Theunissen FE (2009) Functional groups in the avian auditory system. J Neurosci 29:2780-2793. 